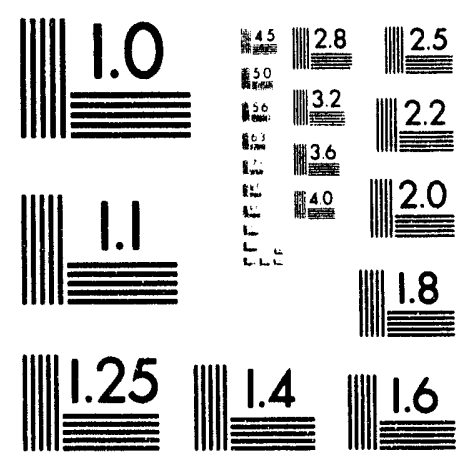



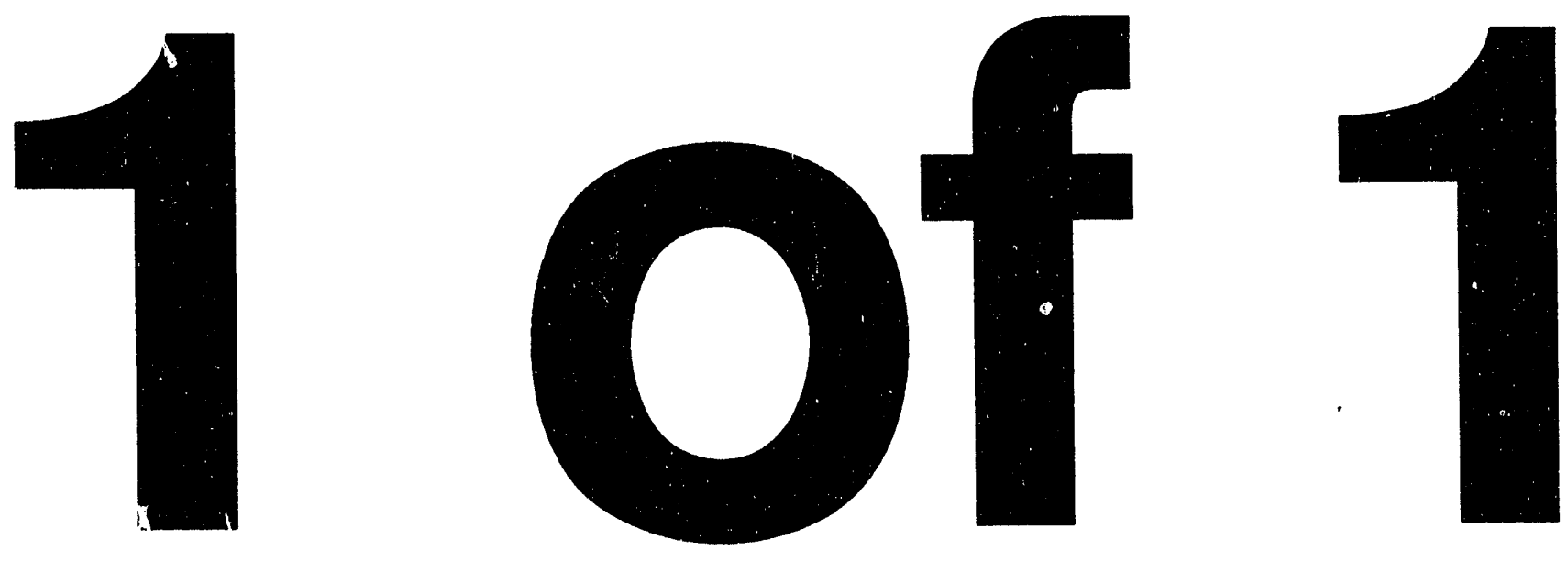
TITLE: DEVELOPMENT OF A NON-LTE SPECTRAL POST-PROCESSOR FOR DENSE PLASMA SIMULATIONS WITH APPLICATION TO SPECTROSCOPIC DIAGNOSTICS IN SPHERICAL IMPLOSIONS AT NOVA

AUTHOR(S): Gregory D. Pollak, X-6, Norman D. Delameter, P-14, Jeffery K. Nash, V-Division, LLNL, Bruce Hanmel, Y-Division, LLNL

\section{DISCLAIMER}

This report was prepared as an account of work sponsored by an agency of the United States Government. Neither the United States Government nor any agency thereof, nor any of their employees, makes any warranty, express or implied, or assumes any legal liability or responsibility for the accuracy, completeness, or usefulness of any information, apparatus, product, or process disclosed, or represents that its use would not infringe privately owned rights. Reference herein to any specific commercial product, process, or service by trade name, trademark, manufacturer, or otherwise does not necessarily constitute or imply its endorsement, recommendation, or favoring by the United States Government or any agency thereof. The views and opinions of authors expressed herein do not necessarily state or reflect those of the United States Government or any agency thereof.

By acceptance of inis article. the publisher recognizes that the U.S Government retains a nunexclusive, royalty-free license to publish or reproduce the published form of this contribution, or to allow others to do so. i i U.S Government purposes.

The Los Alamos National Laboratory requests that the publistier identily this artir,le as work performed under the auspices of the U S. Department of Energy

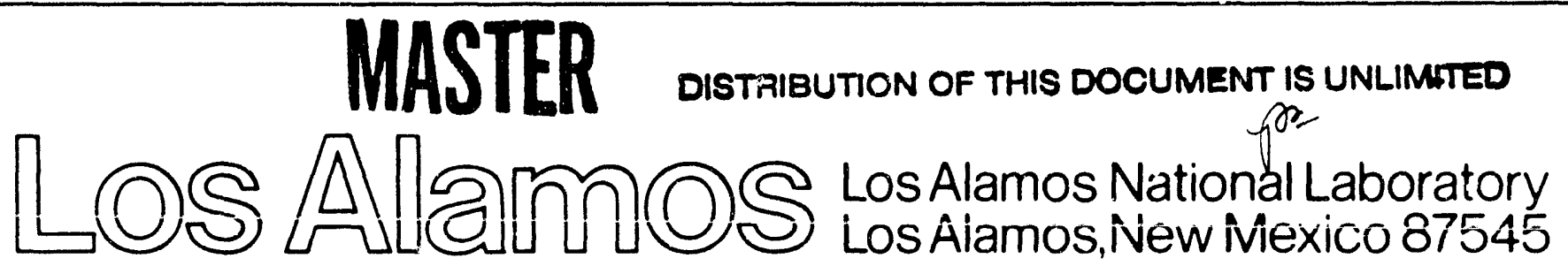




\title{
DEVELOPMENT OF A NON-LTE SPECTRAL POST-PROCESSOR FOR DENSE PLASMA SIMULATIONS WITH APPLICATION \\ TO SPECTROSCOPIC DIAGNOSTICS IN SPHERICAL IMPLOSIONS AT NOVA
}

\author{
by \\ G. D. Pollak, N. D. Delamater \\ Los Alamos National Laboratory \\ J. K. Nash, B. A. Hammel \\ Lawrence Livermore National Laboratory
}

\begin{abstract}
A new non-LTE spectroscopy post-processing package is described. The package processes dump files from 1 or 2-dimensional radiation-hydrodynamics code simulations. Given the grid motion, temperatures, and ion densities contained in the dump files, as well as data from an arbitrarily detailed atomic model, the post-processor calculates internally consistent detailed frequency dependent opacities and radiation fields. The radiation transport equation is solved in the $S_{n}$ approximation using lambda iteration. Sub-cycling is used to achieve a more accurate solution to both the kinetics and radiation field calculations. Line broadening is included using Voigt widths based on the atomic rate coefficients, and Stark widths are included for K-shell spectra. The Sobolev escape factor approximation is available as an option.

This post-processing package has been used to analyze spectra obtained recently at Nova with Ar doped deuterium filled capsules. The dopant was designed to be primarily a density diagnostic (via Stark broadening) but can also be used for temperature diagnosis as well. We have run this post-processor with a wide array of atomic models for Argon, ranging from one containing only singly excited levels for the (important) Hydrogenic, He-like, and Li-like sequences, to one containing a large number of doubly-and triply-excited levels in these sequences. We show a strong dependence of the degree of agreement between simulation and experiment on the model complexity with only the most complex model in close agreement.
\end{abstract}




\section{INTRODUCTION}

Over the last two decades, x-ray diagnostics have become an increasingly important part of moderate-to-high density plasma physics experiments. These diagnostics generally fall into two categories: 2-D imaging instruments, and high-resolution spectrometers. For the imaging experiments, there can be gated time-resolution, but the spectral resolution is achieved by filtering and is, therefore, quite broad. For the spectrometers, the time dependence is achieved by either gating or streaking; and if by gating, then 1-D spatially resolved spectra can also be obtained. Modern spectrometers typically have on the order of 1-eV energy resolution when operating in the 1-5-keV range.

When a simulation of the plasma experiment is carried out using a RadiationHydrodynamics (Rad-Hydro) code, the characteristics of the experimental diagnostics are not usually part of the simulation. The spectral resolution for radiation transport in such simulations is usually quite coarse - typically 20 to 200 bins spread out from $\sim 10 \mathrm{eV}$ to 10 or more $\mathrm{keV}$ (typically logarithmically). This spectral resolution is usually, though not always, adequate for modeling the hydrodynamics and matter temperatures. Often the simulation will use a diffusion algorithm for the radiation transport (especially in 2-D) and it will occasionally do so in plasma regimes where diffusion is not a good approximation, at least for the high energy photons. Finally, in non-LTE regions, the opacities must be calculated in-line in the simulation, and the computational burden associated with this usually limits the kinetics calculations to hydrogenic (principal quantum number only) models. To save additional time, these hydrogenic algorithms are often of an average-atom type, ${ }^{1}$ which collapses the various ionization sequences appreciably present to a single, average, sequence (one for each species). Virtually all of the above algorithms and resolutions are chosen as a trade-off between realism and computational burden.

In order to obtain x-ray diagnostic simulated output, it is then convenient to postprocess the rad-hydro simulations. The Lagrangian hydro grid, species concentrations, opacities, electron temperatures, and electron and mass densities are written to a dump file at selected time points. The post-processor then reads its own input file, which give specifications concerning the detector(s) (angle w.r.t. the target, distance to target, pixel size and number (spatial and temporal resolution), frequency and time response, gating, filtering, etc.). The post-processor then calculates the absorption and emission of $\mathrm{x}$ rays along a line-of-sight that passes through the plasma to a pixel on the detector surface, for a variety of frequencies, for each time point on the dump file. By simulating the detector output in this post-processing mode, it is possible to change specifications concerning the detectors without redoing the rad-hydro run. More importantly though, it is possible to 
relax certain modeling assumptions (the diffusion approximation, the opacity modeling) or the course resolution in frequency where such approximations do not appreciably affect the rad-hydro simulation but do affect the detector simulation. This is an especially important issue for spectrometer simulations, and it is the driving reason for developing the postprocessing capabilities discussed in the next sections.

\section{TDG/DCA-A DETAILED CONFIGURATION ACCOUNTING X-RAY DIAGNOSTIC POST-PROCESSOR}

\section{A. Original Capabilities of TDG}

TDG is a time-dependent post-processor that was originally written approximately 20 years ago, and has since been enhanced in various ways. It was four primary approximations.

The first is that the radiation that impinges on a ziven pixel is solely due to radiation traveling along a line-of-sight (LOS) perpendicular to the detector surface. This is equivalent to the statement that each pixel has 0 . acceptor e angle. The second is that only rays which intersect the rad-hydro grid are processed. Thus, rays coming directly from a backlighter (without hitting the plasma) are ignored. Finally, the third is related to the first two. When computing spatially integrated $x$-ray fluences, the intensity must be multiplied by an area and a solid angle. The solid angle used is the ratio of the same area factor divided by the square of the distance between target and detector. The use of the same area factor in both places (given by the area of an individual pixel) follows from the first two assumptions. The quantitative assessment of these approximations is difficult to consider without actually constructing a code which does not make these approximations. However, for the spatially integrated self-emission spectroscopy simulations discussed later in this article, the approximations are likely to be quite valid.

The intensities along a given LOS ray are computed via the formula:

$$
\begin{gathered}
I(A, \nu)=B L(\nu) e^{-z k_{v}}+\int_{0}^{z} S_{\nu}\left(z^{\prime}\right) . \\
e^{-(z-z \prime) k_{\nu}} d z^{\prime}
\end{gathered}
$$

with 


$$
\begin{aligned}
\nu & =\text { Frequency } \\
z & =\text { Distance from backside of target to detector surface } \\
k_{\nu} & =\text { Absorption opacity } \\
S_{\nu} & =\frac{\epsilon_{\nu} B_{\nu}}{k_{\nu}} \\
B_{\nu} & =\text { Planck function } \\
\epsilon_{\nu} & =\text { Emission opacity } \\
B L(\nu) & =\text { Backighter function }
\end{aligned}
$$

This standard result is the solution to the time independent radiation transport equation along a characteristic direction (LOS). It automatically gets the free streaming and the diffusion limits. Thus, even in the original TDG, some improvement in radiation transport has been achieved.

The fact that a time-independent formula is used even for problems where $k_{r}$ and $S_{r}$ are time dependent is the fourth basic approximation. The validity of this approximation is partly based on the fact that dumps are separated in time by an amount appreciably greater than the transit time of a ray through the plasma, as well as the fact that detectors have finite temporal resolution (if streaked) or integration times (if gated) that are typically greater than or equal to the interval between dumps. However, if the plasma dynamics $\left(T_{e}, k_{\nu}, S_{\nu}\right)$ are changing quickly compared to transit times or temporal resolution, then this approximation can still introduce errors.

\section{B. New Capabilities for TDG}

As originally constructed, TDG used $k_{\nu}$ and $\epsilon_{\nu}$ that were calculated by the rad-hydro code (using identical frequency grids as well). The major advance which this article discusses has been the addition of a sophisticated non-LTE kinetics/opacity package in-line into TDG. The packiage is a DCA (Detailed Configuration Accounting) algorithm which uses an arbitrarily detailed atomic model to supply information to the kinetics and opacity packages. But in order for the new opacities to be correct they must use a photon field which is self-consistent with the opacities. Thus, in every zone for which new opacities are desired, a photon field is calculated using DCA opacities calculated on the last "cycle". Since the basic quantities can change appreciably between dumps, the code can subcycle 
the photon field/kinetics/opacity complex of calculations arbitrarily finely at the user's request. In addition, the user can specify an arbitrarily fine frequency grid. Typically, the binning used mimics the frequency resolution of the spectrometer (about $1 \mathrm{eV}$ at energies of $1 \rightarrow 5 \mathrm{keV}$ ). The user also controls which zones are to have their opacities calculated in TDG and which are to have theirs taken from the dump file (typically LTE zones).

In general, the temperatures and electron densities that would be calculated via the new DCA populations and opacities are likely to be different from those calculated by the Average Atom Rad -Hydro opacity package. Therefore, it is theoretically desirable that new temperatures and densities be computed and used within TDG/DCA. However, there is a fundamental issue which curtails the utility of this, and that is the lack of heat conduction within TDG/DCA. This physical process is generally quite important. The code has the necessary coding to use internally calculated $T_{e}$ and $N_{e}$, but because of this issue, this option is essentially never used.

The next four parts discuss the kinetics/opacity and photon field algorithms in more detail.

\section{Kinetics and Opacity Calculations}

The atomic level populations are computed by solving a set of coupled rate equations:

$$
\frac{d Y_{\ell}^{\prime}}{d t}=R_{\ell \ell^{\prime}} Y_{\ell^{\prime}}
$$

The solution procedure is fully implicit in time:

$$
Y_{\ell}(t+\Delta t)=[I-R \Delta t]_{\ell \ell^{\prime}}^{-1} Y_{\ell^{\prime}}(t)
$$

Here, $R_{\ell^{\prime}}$ is a rate matrix which includes contributions from all the usual BB excitation and deexcitation, $\mathrm{BF}$ ionization and recombination, and Auger and Dielectronic recombination processes. In addition, the package has the capability to handle a special kind of "pseudo" Auger process in which a photo-ionization process creates an inner shell vacancy (and thus a multiply excited state) which subsequently decays via either Auger or photodeexcitation. The intermediate multiply excited state is considered to be in steady-state and its population may be explicitly solved for in terms of the various rates into or out of it as well as the initial and final state populations. Thus, the multiply excited level never need be solved for within the coupled rate equation system. 
The DCA package is highly modular so that other, exotic, processes such as double Auger, can be added easily in the future.

The atomic model from which the rates are calculated is generally created externally prior to the TDG/DCA by a suite of atomic physics codes. For any given set of sequences (of bound electrons), these codes typically generate a fully relativistic, fully multiconfigurational set of states and energies up through some specified maximum principal quantum number (PQN), together with all the associated state-to-state rates. The user can then collapse this full set of states and rates to a smaller set by various procedures. The photoionization frequency dependence is fit a standard formula with four parameters. The collisional BB and BF cross sections are numerically integrated against Maxwellian energy distributions at a wide array of temperatures, and the results are then fit to a 4-parameter formula. These various fitting coefficients are then what constitute much of the rate section of the model.

When calculating photo $\mathrm{BB}$ and $\mathrm{BF}$ rates, the frequency dependence of the photon field across any given photon bin (or group) is isot considered constant. Rather it is fit to a dilute blackbody plus a constant. This is important when the binning is coarse as it allows the LTE (Planckian) limit to naturally be achieved. The integrals over a bin for various upward, downward, and stimulated rates can then be done semi-analytically. This feature is generally not important for spectroscopy calculations because of the very fine binning that is used.

A user may request that a simple model be built at the beginning of a run, rather than use an already built model. This model will use screened hydrogenic formulae (with only PQN dependence) for the energy levels, and corresponding simple hydrogenic formulae for the various rates (e.g., Kramer's formula for photoionization, etc.). Typically, this simple model would only be used for elements which do not exhibit BB features (lines) in the spectral window of the detector (such as hydrogen, carbon, oxygen, etc.).

In addition to the combined photon field/kinetics/opacity subcycling previously mentioned, the kinetics is usually sub-subcycled alone. This subcycling is based on changes in $\bar{Z}$ from one cycle to the next and is not user-controllable.

Continuum lowering is included in the calculation only by limiting the maximum PQN present in the model (either detailed or hydrogenic). There is currently no detailed continuum lowering model that would smoothly extinguish a level as a function of density and temperature, but one is planned for the future. 


\section{Line Broadening}

During the computation of the opacities, it is possible to give $B B$ transitions a line profile. For large mod-ls there are typically tens of thousands of $\mathrm{BB}$ photo transitions and it is not computationally feasible or necessary to have a profile for each of these. For that reason, profiles are computed only for those transitions that the user requests. Typically this is done only for lines within the spectrometer window, and only for those that have appreciable Einstein A values. There is a utility program available which can scan the atomic model and extract out the needed information for important lines. For large models, this procedure generates on the order of 500-1000 profiled transitions.

In the current structure for the code, the default profile is a Voigt profile. If the user requests that an escape factor analysis be performed for a given line, then the profile will be determined by this analysis (see Part $F$ below). In future versions of the code, the Stark broadening (see below) for the lines will not necessarily be included as a component of the Lorentz width of a Voigt profile (as is now done). Rather, an option will be available that computes the total Stark profile for a complex of line (including satellite lines) using algorithms of varying levels of sophistication.

The Voigt parameter is essentially the ratio of the Lorentz width to the Doppler width. The Lorentz width is nominally the sum of the Lorentz widths for the upper and lower levels. The Lorentz width for a level is the sum of all inverse lifetimes (rates) for all processes that deplete the level. These include all of the following:

1. natural inverse lifetime

2. stimulated emission inverse lifetime

3. collisional excitation inverse lifetime

4. photoionization inverse lifetime

5. collisional ionization inverse lifetime

6. Auger inverse lifetime, etc.

In addition to these homogeneous broadening processes, there is additional inhomogeneous broadening that becomes dominant at ion densities above $10^{23} / \mathrm{cc}$ : Stark broadening. As mentioned above, this is currently treated as a width which is added to the standard Lorentz width. The width is calculated via some very simple formulas ${ }^{2}$ that are purely hydrogenic (PQN only) in nature: 


$$
\begin{aligned}
& \Gamma_{S}=D E 1 \text { if ion is hydrogenic } \\
& \Gamma_{S}=\max (D E 1, D E 2) \text { otherwise }
\end{aligned}
$$

with

$$
\begin{aligned}
D E 1 & =F 1 \cdot 4 Z\left[\left(\frac{n_{u}^{2}}{Q_{\ell}}-\frac{n_{\ell}^{2}}{Q_{u}}\right) / R Z+\left(\frac{n_{u}^{4}}{Q_{u}^{2}}-\frac{n_{\ell}^{4}}{Q_{\ell}^{2}}\right) / R Z^{3}\right] \\
D E 2 & =F 1 \cdot 4 Z\left[\frac{3}{2} Z\left(\frac{n_{u}^{7}}{Q_{u}^{3}}-\frac{n_{\ell}^{7}}{Q_{\ell}^{3}}\right) / R Z^{4}+\left(\frac{n_{u}^{4}}{Q_{u}^{2}}-\frac{n_{\ell}^{4}}{Q_{\ell}^{2}}\right) / R Z^{3}\right] \\
F 1 & =\frac{R_{y}}{\left[1+\frac{3}{2} Z \cdot Z R \cdot \frac{R_{y}}{T \cdot R Z}\right]} \\
Z & =\text { average ionic charge of plasma } \\
Z R & =\text { average ionic charge of radiator } \\
R_{y} & =\text { Rydberg energy }=27.2 \mathrm{eV} \\
R Z & =\text { average ionic separation } / a_{o} \\
a_{o} & =\text { Bohr radius } \\
T & =\text { ion temperature } \\
n_{u(\ell)} & =\text { PQN of upper (lower) level } \\
Q_{u(\ell)} & =\text { screened charge of upper (lower) level }
\end{aligned}
$$

These formulas appear to be accurate to considerably better than a factor of 2 , but probably not accurate to better than $10 \%$.

The application of these formulas has been limited to the main lines generated by singly excited states - not to dielectronic satellite lines. This is due to two issues: the fact that the derivation (as such) was predicated on a singly-excited ion; and that the screened charges $(Q)$ are derived from an effective charge Bohr formula:

$$
E_{n}=\frac{R_{y} Q_{n}^{2}}{n^{2}}
$$


where $E_{n}$ is taken from the model and is the energy of the state relative to the ground state of the next (more ionized) sequence. For most multiply-excited states, $E_{n}$ will then be negative and $Q_{n}$ undefined. If the reference energy in the next sequence is taken to be the energy of the state with the electron undergoing (de-)excitation removed (rather than the ground state), then this problem could be avoided. However, this requires a search and state identification procedure that has not been implemented yet. An alternative approach is to use a Mayer screening constant algorithm to compute $Q^{3}$. This procedure will, however, result in $Q$ s somewhat inconsistent with the state energies.

Because of the importance of Stark broadening to much of the spectroscopy being analyzed with TDG/DCA, it is highly desirable to implement more sophisticated procedures than the above. A second level of sophistication is that used in the RATION code. ${ }^{4,5}$ The algorithm used there calculates a Stark profile for individual lines, but still does not deal with dielectronic satellite lines, either singly or as a complex with the main line.

State-of-the-art algorithms are used by two groups. ${ }^{6,7}$ They simultaneously treat all lines in a complex. These codes could conceivably be installed as subroutines in TDG/DCA. Computational time could be a major problem, but since the radiation transport is timeindependent, they coild be turned on only for selected time-slices of interest.

\section{E. Photon Field Calculations}

The kinetics requires a photon field at essentially all frequencies for photo-BF processes, and a $\bar{J}$ (integral of the photon field with the line profile) for photo-BB processes. The algorithm used is a time-independent $S_{n}$ (ray based) procedure (Lambda iteration). This makes it self-consistent with the detector LOS integrations.

A set of rays is defined by (user) specifying an $S_{n}$ order and a type of quadrature set. The usual set is a Chebyshev-Legendre product set which has a total of $N^{2}$ rays on a sphere, where $N$ is an even number. For. each zone that a detailed opacity has been requested, each ray is propagated outward $f_{2} \mathrm{~mm}$ the zone center until it exits the problem mesh. Then it is initialized to any exterior boundary conditions, and propagated back towards the zone center, depositing energy via absorption, and gaining energy via emission according to the same time-independent integral formula used by TDG in calculating detector signals:

with

$$
I(z, \mu)=I_{o}\left(z_{o}, \mu\right) e^{-\left(z-z_{o}\right) \frac{k_{\mu}}{\mu}}+\frac{1}{\mu} \int_{z_{o}}^{z} S_{v}\left(z^{\prime}\right) e^{+(z 1-z) \frac{k_{\nu}}{\mu}} d z^{\prime}
$$

$$
\mu=\text { angle of ray w.r.t. } \mathrm{r} \text { vector or } \mathrm{z} \text {-axis }
$$


These ray-dependent intensities are summed over rays (angles) with appropriate weights to give $J_{v}(z)$. This calculation is repeated for each TDG frequency, including frequencies containing only continuum radiation.

\section{F. Escape Factor Formalism}

When a given BB photo-transition experiences an optical depth appreciably greater than 1. at any frequency in the line profile, the Lambda iteration procedure discussed in the previous section fails to adequately couple the kinetics (and opacities) with the photon field within a single pass or iteration. There are several techniques to correct this deficiency. The simplest is simply to iterate the Lambda calculation approximately once for each increment of 1 . in m:s:m:m optical depth from the preceding dump.

There are several techniques c varying sophistication that are computationally less intensive than the iteration procedure. The simplest of all of these line transfer techniques is a so-called Escape Factor Formalism. The TDG/DCA package has such an algorithm: the specific one used here is due Sobolev ${ }^{8}$ and incorporates the effects of a doppler shift gradient that reduces the optical depth.

The kinetics is altered to provide feedback of the photon field onto the $\mathrm{BB}$ rates and thus populations. Specifically, the BB photoexcitation rates use a photon field given by:

$$
\bar{J}=(1-\operatorname{aesp}) \cdot S
$$

with

$$
S=\frac{n_{u}}{\left(n_{\ell} g_{u} / g \ell-n_{u}\right)}=\text { source function }
$$

and

$$
\text { aesp }=\text { escape probability }=\int_{-\infty}^{\infty} \psi(x) d x
$$


with

$$
\begin{aligned}
\psi(x) & =\left\{\beta+\phi(x) \int_{0}^{1} d \mu \exp \right. \\
& {\left.\left[-\left(1+\frac{\beta}{\phi(x)}\right)\left(-\frac{1}{\gamma \mu^{2}}\right) \int_{x}^{x+\gamma \mu \tau} \phi\left(x^{\prime}\right) d x^{\prime}\right]\right\} /(\beta+\phi(x)) . } \\
\beta & =\frac{k_{c}}{k_{\ell}}=\frac{\text { continuum absorption opacity }}{\text { line absorption opacity }} \\
x & =\frac{\nu-\nu_{c}}{\Delta \nu_{d}} \\
\nu & =\text { Frequency } \\
\nu_{c} & =\text { line center frequency } \\
\Delta \nu_{d} & =\text { Doppler width } \\
\gamma & =\frac{d\left(\nu / \nu_{T h}\right)}{d r} \\
\nu & =\text { optical depth } \\
\nu & =\text { angle cosine relative to radius vector or } \mathrm{z} \text { axis } \\
v_{T h} & =\text { thermal velocity. } \\
x) & =\text { Voigt profile } \\
\tau & \\
\nu &
\end{aligned}
$$

The optical depth can be calculated by either of two techniques. In the first (and easiest) approach, the user inputs a universal distance that is then multiplied by $k_{\ell}\left(\nu_{c}\right)$. The second approach involves calculating 2-column densities of populations for each level involved in an escape factor transition: one column density for each direction from the zone in question to the surfaces on either side. This is clearly a one-dimensional (1-D) concept. In fact, the standard Sobolev formalism given above is only applicable to 1-D slab or (with minor modifications), 1-D spherical geometries. 2D problems are not suitable for escape factor analysis. 
The effects of escape factors on the opacities (as opposed to the kinetics) can be incorporated by using

$$
\frac{\chi(x)}{\int \chi(x)}
$$

instead of $\phi(x)$ for the line profile.

The escape factor approach is implemented on a line-by-line basis by user request. For any lines so requested, the escape factor $\bar{J}$ and $\chi(x)$ are used in place of the Lambdaiteration-calculated $\bar{J}$ and $\phi(x)$, respectively.

\section{SPECTROSCOPIC MODELING APPLIED TO NOVA EYPERIMENTS}

\section{A. Overview of Experiments}

In the Inertial Confinement Fusion (ICF) programs in the U.S. and abroad, a standard technique for investigating various aspects of the implosion dynamics is to dope the fuel (DD or DT) or pusher with small amounts of mid-Z elements that strip down to the K-shell at some point in the implosion. A time resolved, spatially integrating crystal spectrometer is then used to view the plasma at frequencies where the $\mathrm{H}$-like and He-like lines, together with their satellites, will occur. ${ }^{9,10}$

The primary purposes of these spectroscopy experiments are to determine DD and pusher density (from Stark broadening) and temperature (from line intensities) time histories near the time of maximum compression. K-shell spectroscopy is used because the major jump in ionization energies between $\mathrm{K}$ and $\mathrm{L}$ shell electrons produces high energy photons that pass through the pusher, and because of the comparative simplicity of the spectra. The latest experiments at the ICF facility (NOVA) at Lawrence Livermore National Laboratory (LLNL) have used a dopant of Ar present at a molar concentration of .1\% in the DD capsule section.

TDG/DCA has been used to model the observed Ar spectrum. A variety of different atomic models of increasing complexity have been tried in attempting to match the data as close as possible.

There is a fairly wide variation among experimental spectra. Most of the variations between capsules appear to be due to three main issues: unintentionally nonsymmetric drive due to variations in energy and laser profiles among the ten NOVA laser beams; variations in total drive energy; and variations in shell thickness away from nominal. For the purpose of this paper, we have focused our attention on a "standard" capsule having a reasonably 
spherical drive with a roughly nominal total drive energy and shell thickness. However, in order to illustrate the kinds of variations in spectra these capsule/drive variations can induce, some modeling results for a "colder" drive and larager shell thickness are presented as well. All computer runs reported in this paper are 1-D, although 2-D runs have been made for nou spherical implosions.

\section{B. Description of the Atomic Models}

A total of five models have been run in TDG/DCA. In order of increasing model size, the following driscriptions indicate the level structure for all sequences present in the models.

Models 2 through 5 ave fully relativistic level structure for sequences $\mathrm{H}$-like through Be-like. All models contain the (single level) bare sequence.

Model 1 (70 levels): non-relativistic mostly hydrogenic (PQN only) model. Up through $n=10$ for H-like, Fie-like, $n$ nd Li-like singly excited (S.E.) levels. Six doubly excited (D.E.) $2 \ell 2 \ell$ levels in each of $\mathrm{F}$-like and Li-like sequences (some angular momentum splitting). Ground states for Be-like through Ar-like. This model was generated by the RATION code.

Model 2 (183 levels): Up through $n=4$ S.E. For H-like through Be-like. No D.E. Helike. $2 \ell 2 \ell^{\prime}$ and $2 \ell 3 \ell^{\prime}$ D.E. Li-like. $2 \ell 2 \ell^{\prime}$ and some $2 \ell 3 \ell$ D.E. Be-like. $n=2,3$, and 4 S.E. (PQN only) levels for B-like through N-like.

Model 3 (288 levels): Same S.E. H-like through Be-like as in model 2. $2 \ell 2 \ell^{\prime}$ D.E. He-like. $2 \ell 2 \ell^{\prime}, 2 \ell 3 \ell^{\prime}, 3 \ell 3 \ell^{\prime}$ D.E. Li-like. $2 \ell 2 \ell^{\prime}$, some $2 \ell 3 \ell^{\prime}$, and $2 \ell 4 \ell^{\prime}$ D.E. Be-like. $2 \ell 2 \ell^{\prime}, 2 \ell 2 \ell^{\prime} 2 \ell^{\prime \prime}$ D.E. and triply excited (T.E.) levels in B-like (jj-averaged). Similar levels in C-like and N-like.

Model 4 (609 levels): Up through $n=5$ S.E. H-like through Be-like. $2 \ell 2 \ell^{\prime}, 2 \ell 3 \ell^{\prime}, 2 \ell 4 \ell^{\prime}$, $3 \ell 3 \ell^{\prime}, 3 \ell 4 \ell^{\prime}$ D.E. Li-like. Similar Be-like structure to model 3. Same B-like through N-like structure as in model 3.

Model 5 (1380 levels): Same S.E. structure for H-like through Be-like as in model 4. $2 \ell 2 \ell^{\prime}, 2 \ell 3 \ell^{\prime}, 2 \ell 4 \ell^{\prime}, 3 \ell 4 \ell^{\prime}$, and $4 \ell 4 \ell^{\prime}$ D.E. for both He-like and Li-like. $2 \ell 2 \ell^{\prime} 2 \ell^{\prime \prime}, 2 \ell 2 \ell^{\prime} 3 \ell^{\prime \prime}$, $2 \ell 2 \ell^{\prime} 4 \ell^{\prime \prime}$ triply-excited (T.E.) levels in Li-like. Same Be-like through $N$-like structure as in model 4. 


\section{Comparison of Experimental and TDG/DCA Spectra}

The results presented in this paper represent the first phase of a detailed study of the NOVA Ar spectra. In this phase the emphasis has been on converging the atomic model. At first this was considered to be possible with a relatively small number of levels, but experience has indicated otherwise. This can best be illustrated by looking at the ratios of the 4 main line complexes that the spectrometer usually has in its frequency window. In order of increasing energy, these line complexes are: $\mathrm{He} \alpha(1 \rightarrow 2), \mathrm{H} \alpha(1 \rightarrow 2), \mathrm{He} \beta(1 \rightarrow 3)$ and $\mathrm{H} \beta(1 \rightarrow 3)$, where the prefix He refers to He-like, and $\mathrm{H}$ to H-like. Each complex consists of 2 main lines plus a (generally) substantial number of satellite lines. In this paper, only results for the spectra at maximum Ar ernission (near maximum compression) will be presented. Figures 1 and 2 give examples of experimental spectra. Important, reasonably reproducible, aspects of these spectra are that the $\mathrm{He} \beta$ complex has the maximum amplitude, while the $\mathrm{He} \alpha$ is less than the $\mathrm{H} \alpha$ line. The $\mathrm{H} \beta$ complex is very broad. Figures $3,4,5,6$, and 7 present theoretical spectra from TDG/DCA using models 1 through 5 , respectively. In all cases the absolute numbers on the $y$-axis are arbitrary, since the spectrometer has not been absolutely calibrated. It should be noted that these theoretical curves do not have either the rocking curve of the crystal or the relative spectral response across the window incorporated into the analysis (the latter has only very recently been measured). Hopefully, these issues will not appreciably change the results.

The first thing that is noticeable about the results is the large variation in line ratios for different models. Model 1 (the RATION model) gives very large $\alpha$ to $\beta$ ratios. This improves somewhat with model 2, but the $\alpha$ lines are still much too large. The introduction of $3 \ell 3 \ell^{\prime}$ D.E. levels in going from model 2 to model 3 clearly decreases the $\mathrm{He} \alpha$ to $\mathrm{H} \alpha$ ratio. It was this change in spectra with model complexity that was the first solid indication that the model was not converged, since the same atomic physics codes were used to generate models 2 and 3 (as opposed to model 1). The addition of $n=5$ S.E. levels and $2 \ell 4 \ell^{\prime}$ and $3 \ell 4 \ell^{\prime}$ D.E. Li-like states further decreases both $\alpha$ lines relative to $\mathrm{He} \beta$, with the major effect coming in the He $\alpha$ line. Finally, the addition of $2 \ell 2 \ell^{\prime}, 2 \ell 3 \ell^{\prime}, 2 \ell 4 \ell^{\prime}, 3 \ell 3 \ell^{\prime}, 3 \ell 4 \ell^{\prime}$, and $4 \ell 4 \ell^{\prime}$ D.E. levels for He-like, $4 \ell 4 \ell^{\prime}$ D.E. levels, and $2 \ell 2 \ell^{\prime} 2 \ell^{\prime \prime}, 2 \ell 2 \ell^{\prime} 3 \ell^{\prime \prime}$, and $2 \ell 2 \ell^{\prime} 4 \ell^{\prime \prime}$ T.E. levels to Li-like in model 5 increases $\mathrm{H} \alpha$ relative to $\mathrm{He} \alpha$.

All of the runs of Figs. 3 through 8 were done with no lines requested to have escape factors computed. The optical depths of the $\alpha$ lines are on the order of 10 , so it would potentially be useful to use escape factors for these 2 lines. Such runs have been done, but no significant changes were observed compared to Fig. 7. This is undoubtedly due to the gradual fashion (over many dumps) that the lines increase in optical depth. 
Figure 8 gives the spectra for a TDG/DCA run using the same Model 5 as in Fig. 7 but using dumps from a slightly different Rad-Hydro run. Specifically, the run had a slightly thicker shell (Pusher); it was run using diffusion radiation transport instead of $\mathrm{P}_{n}$; and its drive energy was somewhat lower. The result for the theoretical spectra is quite different from the nominal run. It is not clear at this point which of these 3 issues is the most important determinant in the difference-an ongoing study is attempting to sort this out. Clearly, however. this result indicates that the Rad-Hydro code may generate subtle shifts in temperatures and densities that have decidedly non-subtle effects on spectra.

Comparing Fig. 8 with Fig. 1 indicates that model 5 is generally getting reasonably good agreement with the particular shot of Fig. 1 and very good agreement with the particular shot of Fig. 2. The $\alpha$ lines are somewhat too low relative to $\mathrm{He} \beta$ in comparison to Fig. 1. In addition, there are clearly gaps in the centers of the $\mathrm{H} \alpha$ and $\mathrm{H} \beta$ complexes that are undoubtedly due to a lack of satellite lines with spectator electrons having PQN greater than 4. (Estimates for the maximum PQN present in a plasma at $1 \mathrm{keV}$ electron temperature and $1 \times 10^{24}$ ion density indicate up to $n=10$ (for low $\ell$ values) for H-like, and up to $n=8$ (for low $\ell$ values) for He-like, will be present). The lack of stark broadening for dielectronic satellites wi"' also affect the degree of agreement.

A fundamental issu ration ty the variations in spectra as a function of model cornpleteness, is a precise assessment of what in the models is causing it. To help determine this, an essentially complete set of edits has been installed in the package. These include snapshots and timeplots of individual transition rates, level populations, level potential energies, and population fluxes between levels. The latter 3 quantities can be summed over groups of levels. Although it wasn't possible to analyze all of the runs with these edits by this time, a preliminary edit analysis has been done for Model 5. The primary conclusion to be gained from the edits so far obtained is the importance of the collisional $n=2$ to $n=3$ population flux, for both He-like and H-like sequences. This flux essentially dominates everything else in the problem. If it were not for this upward collisional flux, the $\beta$ lines would actually be in absorption, rather than emission, for many of the DD gas zones. Since the energy differences between $n=3$ and $n=2$ levels are on the order of $600 \mathrm{eV}$, any changes in temperatures in this energy vicinity are likely to substantially shift the spectra. A more complete edit analysis is currently in progress.

\section{Conclusions and Future Work}

The most important conclusion to be drawn from the work so far is the reasonably wide variability in spectra with model completeness, with only the most complete model in 
close agreement with experiment. A second conclusion is the variability with Rad-Hydro run changes, where these changes are standard design issues that induce only relatively minor changes in wide-band $x$-ray images, or neutron emission characteristics. This means that the spectra can be used as sensitive gauges of plasma dynamics, provided that a complete atomic model with accurate rates can be assembled. The third conclusion is that relatıvely good agreement with an experiment has been obtained, but that there is a fair amount of variability between shots that makes any firm conclusions concerning densities and temperatures unwarranted at this time. Given that the spectra are still changing between Models 4 and 5, it is impossible to be certain that models more complete than \#5 will not also change the major line ratios.

There are thus a number of directions for future work. One is to assemble a more complete model, one that has S.E. and D.E. levels with $n \geq 5$ (up to at least 8 ), and more triply excited levels (especially those near the $\mathrm{H} \beta$ line). The studies on drive strength, shell thickness, and radiation transport options within the Rad-Hydro code need to be completed. The analysis of the edits for different models has to be completed. A longer term project is to understand the temperature and density spatial variation as a function of spectral output. This task can be considerably simplified if the spectra are generated from kinetics in quasi (or instantaneous) steady state.

Future work in code development includes better Stark broadening and some continuum lowering options. In addition, versions for workstations (1D) and massively parallel computers (2D) would be useful. Procedures for approximately handling D.E. levels created by other than inner shell processes, where the levels are in quasi-steady state (i.e., tracking the ground state population) would reduce the computational times tremendously.

\section{REFERENCES}

1.) G. Pollak, "Detailed Physics of XSN-U Opacity Package," Los Alamos National Laboratory report LA-UR-90-2423.

2.) H. Griem, private communication.

3.) H. Mayer, "Methods of Opacity Calculations," Los Alamos National Laboratory Report LA-647 (1948).

4.) R. W. Lee, "Spectral Line-Broadening Codes for Hydrogen-, Helium-, and Lithium-Like Ions in Plasmas," Lawrence Livermore National Laboratory Report UCID-21292. 
5.) R. W. Lee, B. O. Witten, J. E. Strout III, JSQRT 32, 91 (1984).

6.) A. Calisti, R. Stamm, B. Talin, and R. Lee, Phys. Rev. A42, 5433 (1990).

7.) L. Woltz and C. F. Hooper, Phys. Rev. A38, 4766 (1988).

8.) V. Sobolev, Soviet Astron. 1, 678 (1957).

9.) B. A. Hammel, et al, "K and L Spectroscopy of Indirectly Driven Implosions," Rev. Sci. Instrum. 63(10), 5017 (1992).

10.) B. A. Hammel, et al., "High Z X-ray Spectroscopy of Laser-Imploded Capsules," Rev. Sci. Instum. 61 (10), 4776 (1990). 


\section{FIGURE CAPTIONS}

Fig. 1. Experimental capsule spectra at maximum emission, Shot A.

Fig. 2. Experimental capsule spectra at maximum emission, Shot B.

Fig. 3. Theoretical spectra, Model 1.

Fig. 4. Theoretical spectra, Model 2.

Fig. 5. Theoretical spectra, Model 3.

Fig. 6. Theoretical spectra, Model 4.

Fig. 7. Theoretical spectra, Model 5.

Fig. 8. Theoretical spectra, Model 5 , variation with hot drive. 


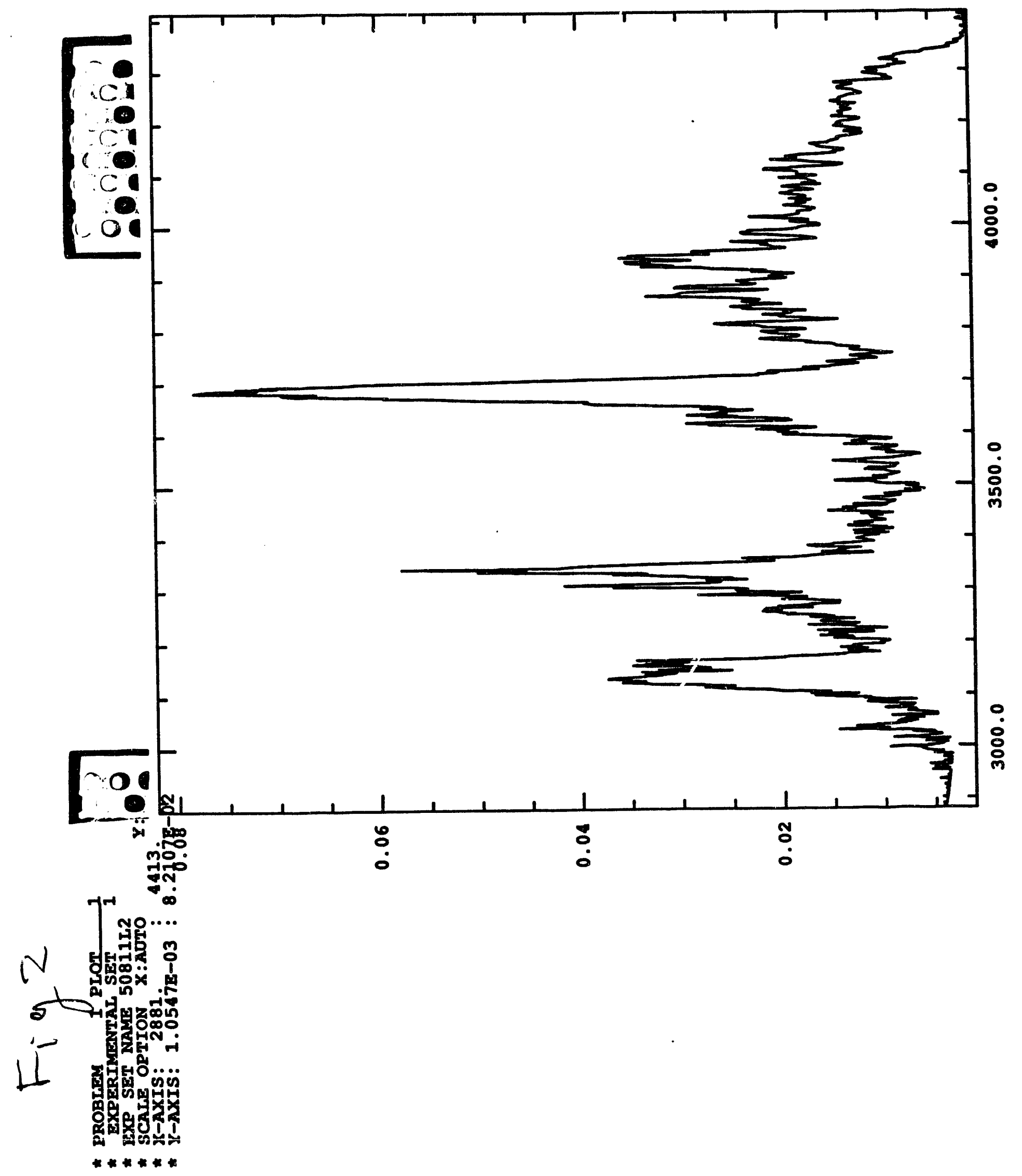




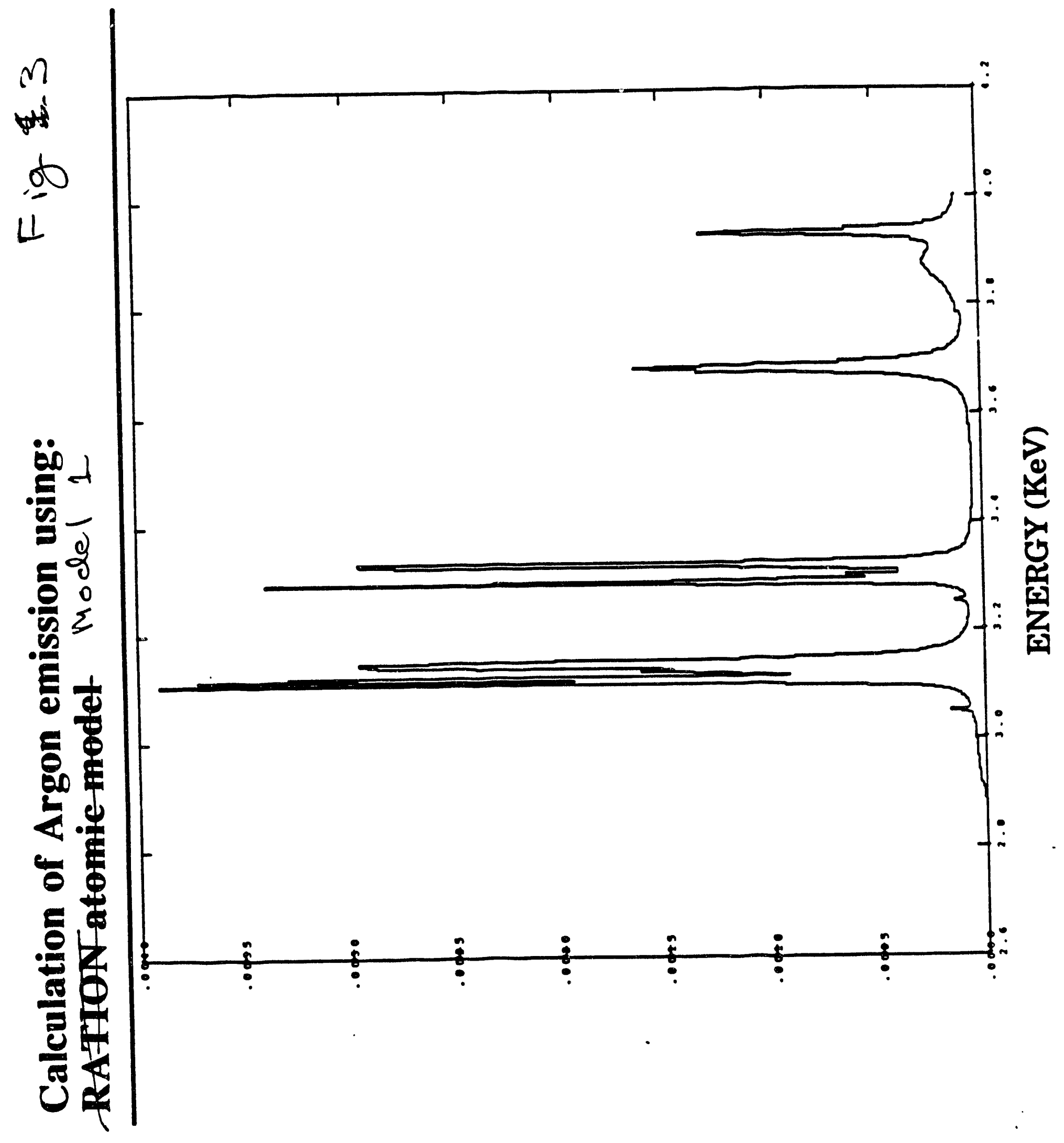




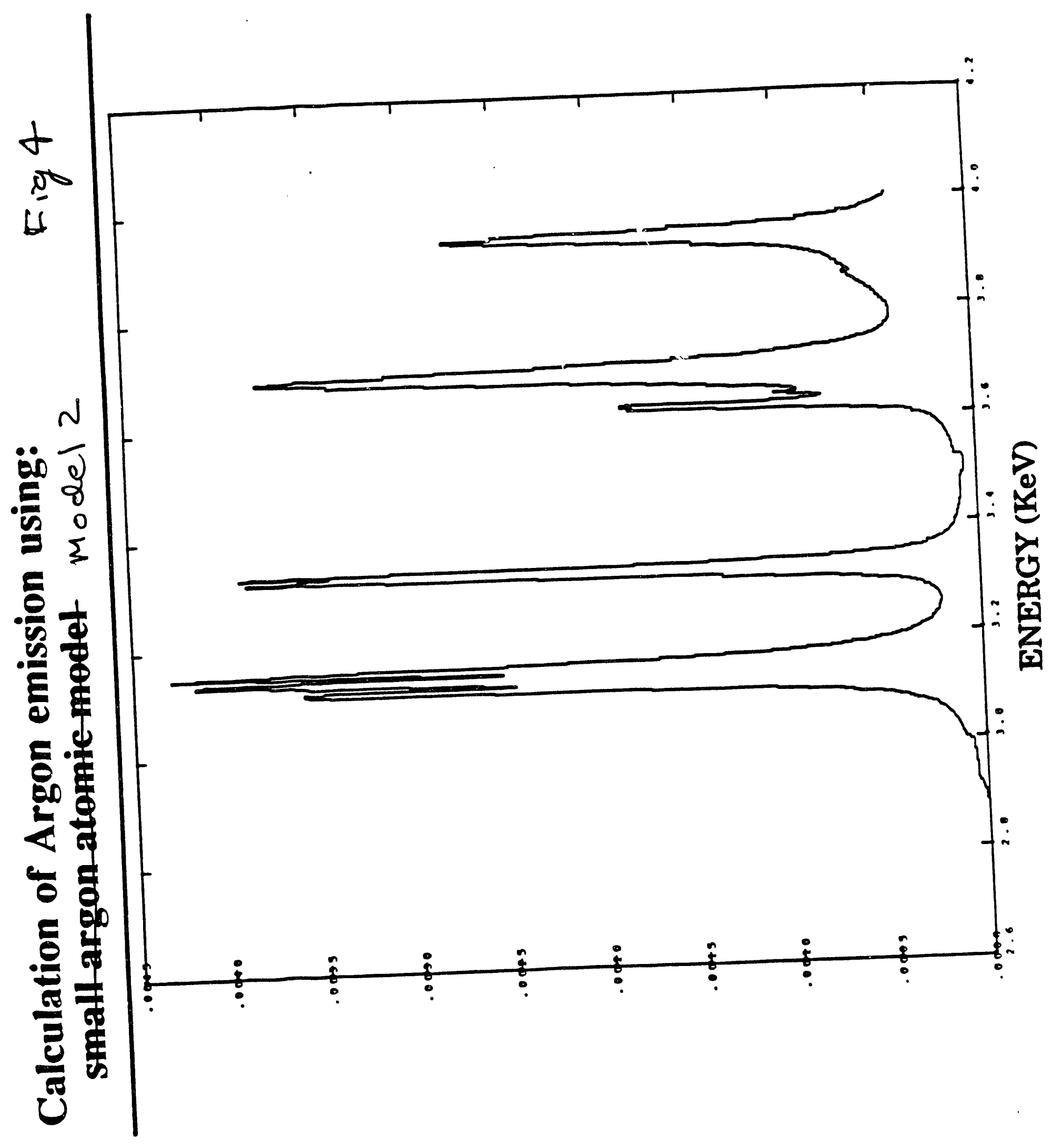


b

ot

I

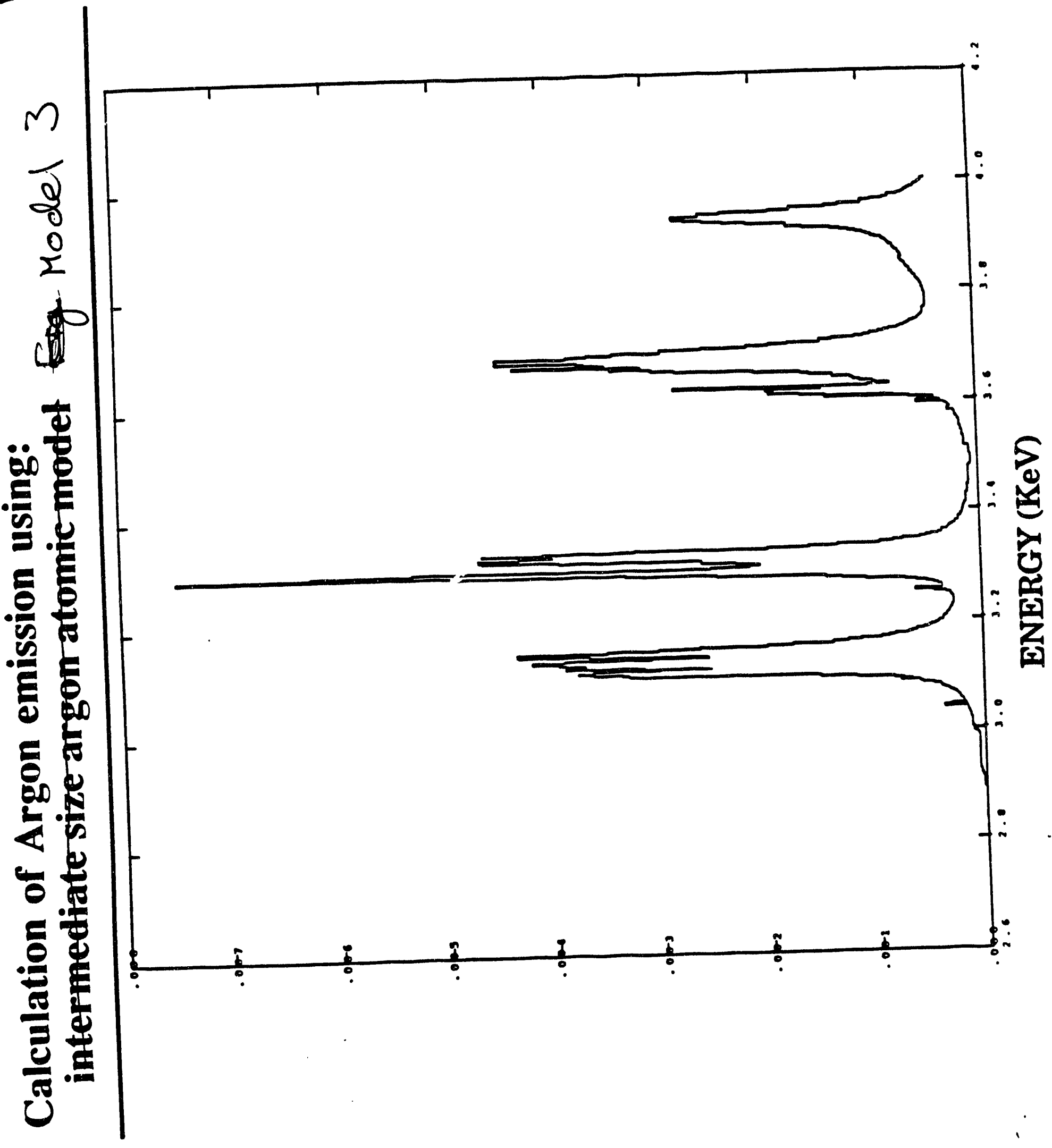


6. ot

t)

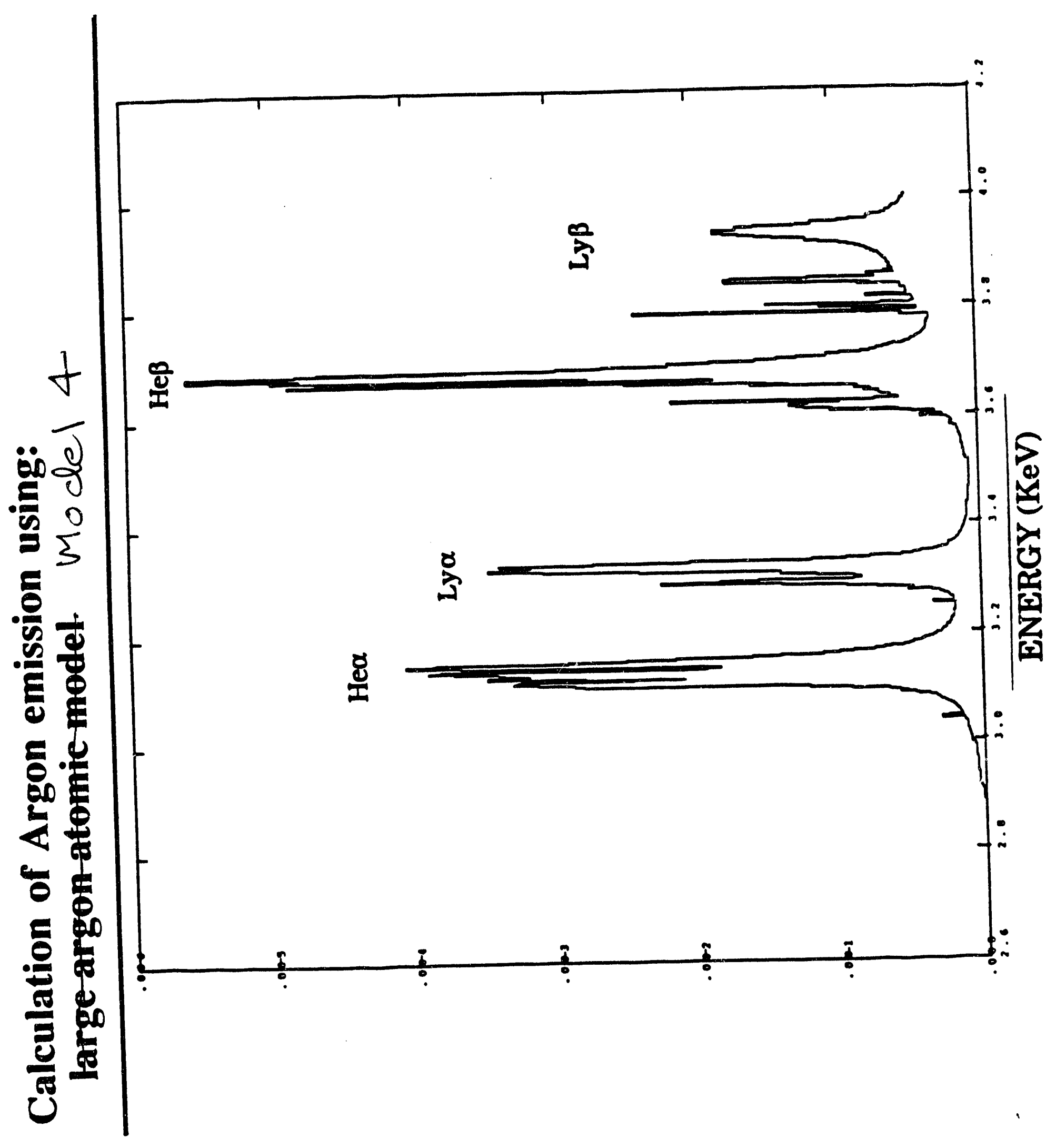




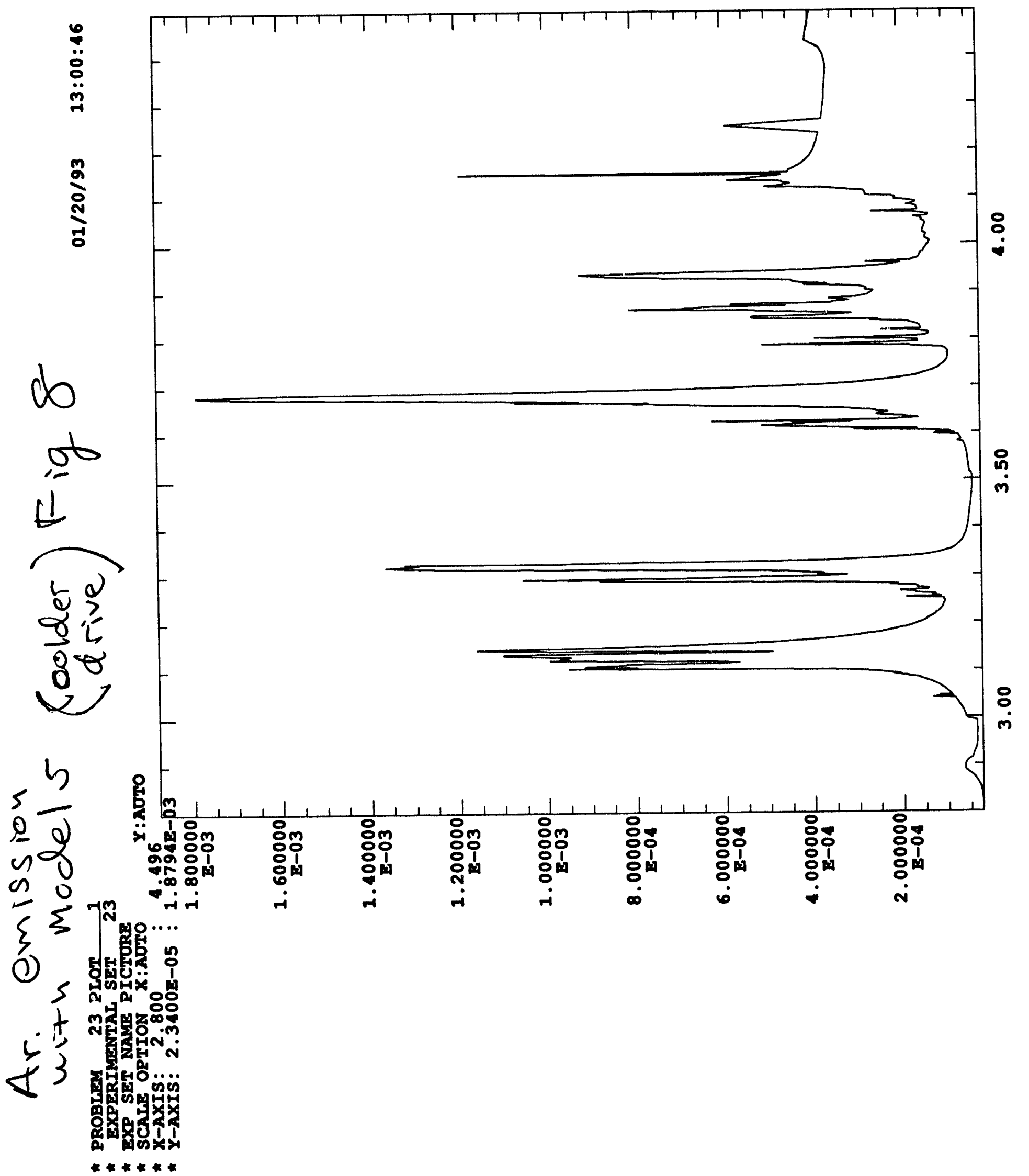



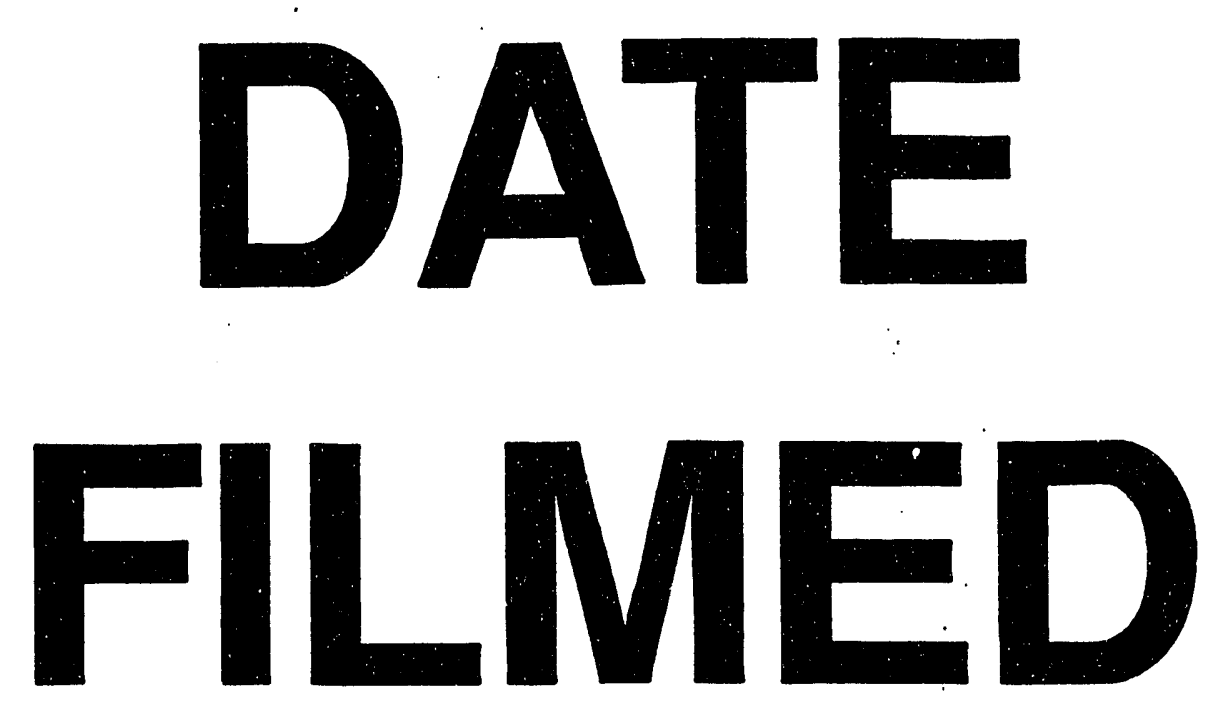

$10 / 13 / 93$
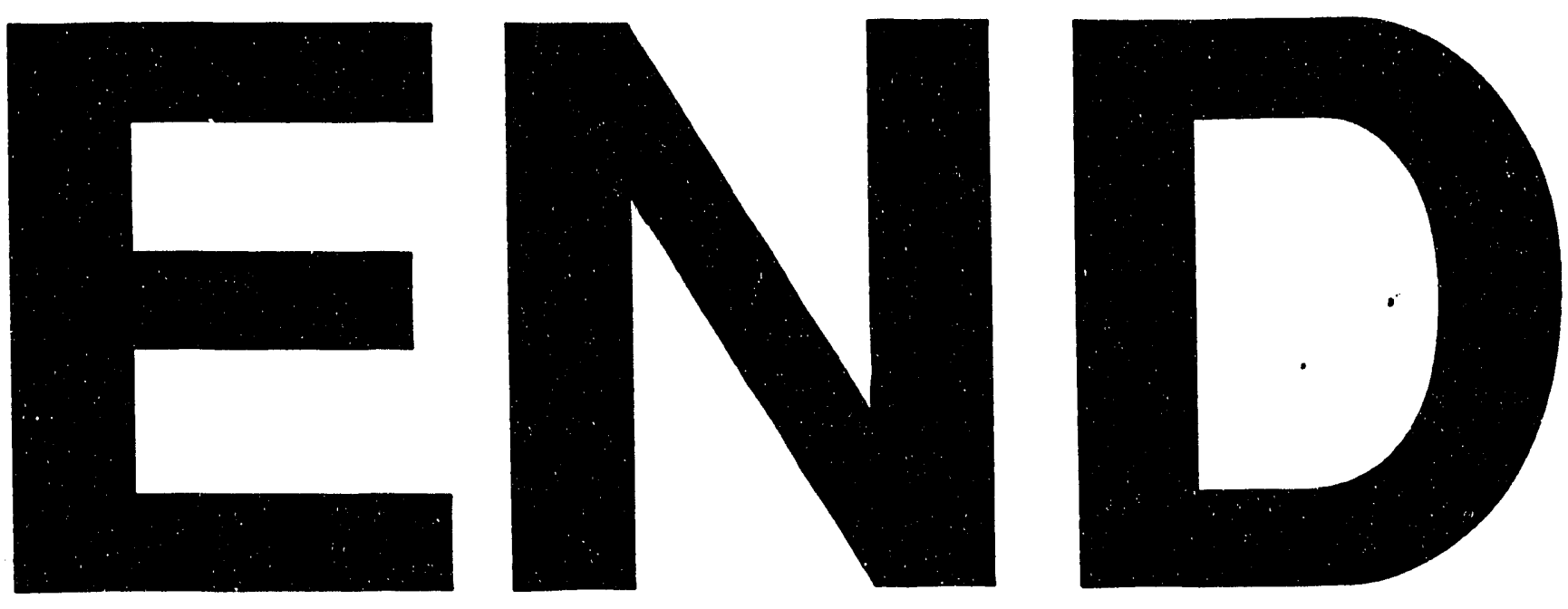
